\title{
Un necrologio flaubertiano e un mazziniano dimenticato
}

\section{Adriana Santoro}

\section{(2) OpenEdition}

1 Journals

\section{Edizione digitale}

URL: https://journals.openedition.org/studifrancesi/37076

DOI: 10.4000/studifrancesi.37076

ISSN: 2421-5856

\section{Editore}

Rosenberg \& Sellier

\section{Edizione cartacea}

Data di pubblicazione: 15 décembre 2004

Paginazione: $547-554$

ISSN: 0039-2944

\section{Notizia bibliografica digitale}

Adriana Santoro, «Un necrologio flaubertiano e un mazziniano dimenticato», Studi Francesi [Online],

144 (XLVIII | III) | 2004, online dal 30 novembre 2015, consultato il 08 mai 2021. URL: http://

journals.openedition.org/studifrancesi/37076; DOI: https://doi.org/10.4000/studifrancesi.37076

\section{(c)}

Studi Francesi è distribuita con Licenza Creative Commons Attribuzione - Non commerciale - Non opere derivate 4.0 Internazionale. 


\section{Un necrologio flaubertiano e un mazziniano dimenticato}

Nel corso di una ricerca riguardante i riflessi italiani dell'opera di un grande romanziere francese dell'Ottocento, può capitare di imbattersi in scritti che, pur non essenziali ai fini del lavoro in questione, forniscano notizie coeve tali da suscitare viva attenzione.

È quanto accaduto a noi con L'Illustrazione Italiana del Maggio 1880. Il giorno 8 di tale mese, un sabato, era venuto a mancare in Normandia Gustave Flaubert: il corrispondente della rivista, D. A. Parodi, dedica gran parte del proprio ampio Corriere di Parigi all'illustre defunto, esprimendosi sull'uomo e sull'artista secondo quanto riportiamo in stralci:

\section{GUSTAVO FLAUBERT}

La Francia ha un grande scrittore di meno: Gustavo Flaubert è morto!

È morto sabato scorso nella sua proprietà di Croisset, ov'egli soleva passare quasi tutto l'anno.

Croisset è un casale vicino a Rouen, la città natale del Corneille, di cui la sera di venerdi egli aveva riletto non so quale tragedia a un medico che, la mattina appresso, chiamato e accorso in fretta, non ritrovò più nell'amico lasciato sano e allegro che un cadavere. Tanto fu improvvisa e quasi istantanea la morte!

Lo uccise, dopo il suo bagno quotidiano e all'uscir di tavola, un colpo apoplettico veramente fulminante. Non aveva che 58 anni.

Egli era sanguigno di temperamento, acceso in viso, grande e massiccio. Aveva gli occhi cilestri e rotondi, simili a due globi di vetro, la barba e $i$ capelli grigi che usava portare lunghi, cadenti sulle spalle atletiche. Copriva la testa voluminosa con un cappello di seta nera basso di forma e largo d'ali, che metteva a sghembo appoggiato sull'orecchio. Non aveva a schifo i buoni bocconi, i vini generosi né, credo, le belle donne; era un buon compagnone e scherzava e dava la baia volentieri, un po' amaro e lubrico nelle sue facezie. Aveva ereditato da Teofilo Gautier, uno de' suoi maestri, l'amore dello stile pittoresco e l'odio del bourgeois a cui dava ogni sorta di qualifiche ridicole o vituperevoli. Era, in somma, un materialone guidato, anche negli studi, dalla sensualità. Libava leggendo, componendo, parlando, pensando. Epperciò era cosi lento nello scrivere, che Madame Bovary gli costò otto e Salammbô sei anni di voluttuosa fatica. Impiegava tutta una mattina per disegnare, colorire, modulare una frase e un'altra per rivederla, contemplarla, goderla trascritta nel bel mezzo di un gran foglio bianco. Lavorava, come l'altro suo mae-

(1) D. A. PARODI «Corriere di Parigi - Gustavo Flaubert», in L'Illustrazione Italiana, VII, n. 20, 16

maggio 1880, pagg. 318-319. 
stro Balzac, in uno stanzino ermeticamente chiuso e, in pieno giorno, a lume di candela. Che cosa avrebbero detto $i$ Greci, che del sole aveano fatto il Dio dell'ispirazione? $O b$ quanti amminicoli e quanto sforzo per produrre un po' di buona prosa!

Quella del Flaubert è delle migliori, ma sente l'olio e, come quella del Gautier, somiglia più a un'agata che a un diamante: ha più solidità e colore che luce. Io non ci sento il calor fecondo e la spontaneità della vita: da ciò la noia che spesso l'accompagna e rende tanto laboriosa la lettura della Tentation de Saint-Antoine, dell'Education sentimentale $e$ di Salammbô.

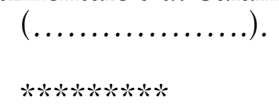

Oh benedetta miopia dei contemporanei, che ci porge, insieme a qualche gustosa "trouvaille", giudizi tali da far oggi inorridire, dopo oltre un secolo di studi e ricerca, lo sterminato stuolo dei flaubertisti... Ma la corrispondenza da Parigi continua:

Io trovo inutile di ritessere l'elogio del suo capolavoro: Madame Bovary. Chi non l'ba letto? e chi non conosce il bello studio che ne ha fatto Sainte-Beuve?

Non ebbi l'onore di vedere che una sola volta il Flaubert, nella casa di una donna gentile, e dopo un lauto pranzo che non aveva smorzato l'estro del bell'umore. Mi riusci molto simpatico, e mi ricordò un italiano morto a Parigi quattr'anni $f_{a}(. .$.

Nell'aspetto infatti e nell'indole dell'ingegno, l'illustre scrittore francese arieggiava Lizabe Ruffoni, il quale, ove non si fosse per sua sventura impacciato di politica, avrebbe dato all'Italia, come quegli alla Francia, un insigne scrittore di più. E benché l'abbia mal servita, egli però l'amava sinceramente, la sua patria, e, occorrendo, ne difendeva i diritti e le glorie con eloquente ardore, con affezione tutta figliale.

Esule a Parigi, ove mori come visse, povero, ei non cessò mai dal rileggere le opere gloriose dell'ingegno italiano che riviveva in lui imaginoso e assennato, arguto $e$ profondo. Pochi libri moderni bo letto che mi abbiano dilettato e istruito come la sua vivacissima e sempre nuova conversazione.(...)

E vero che Lizabe Ruffoni non lasciò di scritto quasi nulla e sprecò in vanissimi colloqui i tesori della sua mente; ma fu meno pigrizia o impotenza che desiderio d'oscurità nato in lui dal rimorso de' suoi falli politici e amore orgoglioso della perfezione. Aggiungi che, al pari del Flaubert, e' leggeva con voluttà, sorbendo a centellini e assaporando ogni bello ed ogni vero senza riuscire ad estinguere la sete della sua intelligenza ch'era quella d'un poeta insieme e d'un filosofo. (...)

$* * * * * * * * * * *$

Lizabe Ruffoni! Chi era costui? A noi, cultori di letteratura francese e segnatamente di Gustave Flaubert e del suo milieu, quel nome non diceva nulla. Ma, interessati e curiosi, ben volentieri ci siamo indotti a deviare dalla nostra strada maestra e ad impegnarci per saperne di più. Consultando quindi, sulla base di quel quattr'anni $f a$, le annate della stessa rivista, ecco in data 12 novembre 1876 l'articolo $^{2}$ che dà notizia della morte di

(...) Lizabe Ruffoni ferrarese. Avanti il 1848 si era dato al mazzinianesimo e fu per lungo tempo segretario dello stesso Mazzini. Rientrato in patria dopo l'amnistia largita da Pio IX ed essendo il futuro triumviro nominato in vari collegii, raccomandò il Ruffoni come suo sostituto nella deputazione di Ferrara, ed egli infatti fu inviato dagli elettori alla Costituente Romana. Caduta Roma, riparò in Francia e continuò per alcun tempo

(2) L'Illustrazione Italiana, III, n. 55, 12 novem- bre 1876, pag. 382 (non firmato). 
a seguire il partito di Mazzini, finché venutogli meno l'appoggio di questi ebbe il torto - che non gli fu mai perdonato - di mettersi al servizio del principe Achille (sic) Murat, che sognava di rimontare sul trono di Gioacchino I. Svanite queste speranze, e fattasi l'Italia, il Rusconi (sic), che nel suo cuore n'era felicissimo, rinunziò alla politica e non si occupò più che di letteratura. Da ben vent'anni gli impiegati della gran biblioteca di via Richelieu lo vedevano comparire ogni giorno e restare immerso fra i libri per parecchie ore consecutive. Non pubblicò nulla sotto il proprio nome, ma chi potrebbe dire quanti si fecero belli delle sue ricerche, e quanti libri furono fatti mediante l'opera sua? Ruffoni era un bel vecchio rubicondo coi capelli e i mustacchi bianchissimi, e una statura quasi colossale; s'incontrava ogni giorno sul boulevard Montmartre, quasi sempre in compagnia di qualche erudito. Pieno di spirito, dotato di ferrea memoria, scettico di carattere, grande linguista, la sua conversazione era preziosissima. E morto di una congestione.

$* * * * * * * * * *$

Notizie dal sapore di attendibilità e in contrasto con una primissima scrematura fatta su enciclopedie, repertori vari, opere sugli esuli politici italiani in Francia, tutti caratterizzati da un assordante silenzio nei confronti del personaggio in questione, del quale si riesce a trovare traccia soltanto in studi assolutamente specialistici. Ecco invece come si esprime l'Archivio Biografico degli Italiani':

LIZABE RUFFONI GAETANO, eletto deputato all'Assemblea Costituente di Roma del 1849, non risulta avere attivamente partecipato ai lavori del Congresso.

Come? Al contrario, il nome di Lizabe Ruffoni si può dire che riempia di sé i verbali delle sedute dell'Assemblea mazziniana fin dal momento in cui egli entra a farne parte quando Giuseppe Mazzini, eletto a Roma e a Ferrara, opta per il collegio dell'Urbe; nella scelta c'è la considerazione di dare a Ferrara la possibilità di essere rappresentata da un suo figlio, il Lizabe Ruffoni, interprete dei sentimenti repubblicani largamente diffusi nella città estense $e^{4}$.

Gaetano Lizabe Ruffoni partecipa ai lavori dell'Assemblea, che vanno dal 5 febbraio 1849 al 27 giugno dello stesso anno, da quando vi prende per la prima volta la parola nella seduta quarantaduesima del 17 aprile ' 49 , con numerosi interventi, sia entrando nel merito della Costituzione che l'Assemblea va elaborando, sia pronunciandosi su temi di politica estera (rapporti con gli Stati cattolici) sia, infine, quando la Repubblica già vacilla sotto la pressione delle armi straniere, per chiedere che vengano puniti con estremo rigore "tutti quei Presidi i quali, mandati in quei luoghi per difendere la patria, non imitano l'esempio dei valorosi che nell'ultimo giorno di aprile fecero dei loro petti un baluardo dinanzi a Roma". La sua fervida e appassionata eloquenza suscita molto spesso uno "scoppio unanime di caldi applausi

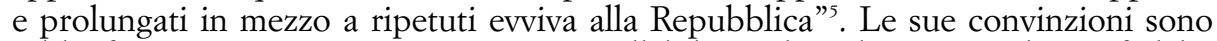
salde, ferrei appaiono i suoi principi, incrollabile sembra al momento la sua fedeltà agli ideali repubblicani.

$* * * * * * * * * *$

(3) Archivio Biografico degli Italiani, Catanzaro, 1998, che riporta integralmente il testo già contenuto in F. ERCOLE, serie XLII, Il Risorgimento italiano, Gli uomini politici, vol. II, in Enciclopedia bio-bibliografica Italiana, Istituto Editoriale Italiano, Roma, 1941.
(4) P. Sanfilippo, Le vicende elettorali di Giuseppe Mazzini, Napoli, 1974, p. 7.

(5) Tutte le citazioni sono tratte dai verbali dell'assemblea Costituente Romana, 5 febbraio 1849 - prima seduta / 27 giugno 1849 - 56 seduta, Roma, senza indicazione tipografica, 1849. 
Gaetano Lizabe Ruffoni si era messo in luce negli anni attorno al '40 negli ambienti degli emigrati politici italiani a Parigi, città dove era giunto in seguito a vicende ancor oggi oscure. Nel 1848 Lizabe Ruffoni è a Milano e figura tra i tredici firmatari, dopo Mazzini, del programma dell'Italia del Popolo, contenuto nel primo numero in data 20 maggio dello stesso anno.

Di Giuseppe Mazzini sono note ad oggi tre lettere a Gaetano Lizabe Ruffoni, tutte connotate da grande stima e fiducia nei confronti del ferrarese, al quale vengono affidati delicati incarichi politici o compiti di propaganda a mezzo stampa ${ }^{6}$.

Sopraggiungono quindi, come si è detto, i mesi di fuoco della Repubblica Roma$\mathrm{na}^{7}$, poi la nuova e questa volta definitiva partenza per la Francia. Qui, pochi anni di attività in seno al movimento repubblicano in esilio, poi l'abbandono dei vecchi compagni per un diverso raggruppamento finché lo stesso Lizabe Ruffoni non sceglierà di tacere, politicamente, per sempre.

Si innesta a questo punto il lato oscuro della vicenda del nostro personaggio, tale da costargli all'epoca roventi polemiche e l'ostracismo da parte dei mazziniani; non solo, ma tale da assegnargli ancora un giudizio piuttosto severo dalla storiografia a noi contemporanea.

A proposito dell'orientamento politico di metà Ottocento che va sotto il nome di "murattismo", riteniamo necessario e sufficiente nell'ambito del presente saggio fare riferimento all'opera di F. Bartoccini ${ }^{8}$ nonché alla relazione della stessa studiosa durante il Convegno Storico Toscano tenuto a Castelvecchio Pascoli nel maggio $1975^{9}$.

Basteranno quindi alcuni accenni ad un fenomeno che se fu "piccola cosa"10 nella realtà, ebbe ben altra immagine agli occhi dei contemporanei, un'immagine che all'epoca agì come elemento concreto e attorno a cui si sviluppò un larghissimo dibattito in quel decennio che, come sembra, fu ben altro che una convinta e serena preparazione alla soluzione unitaria sabauda e cavourriana ${ }^{11}$.

Soprattutto nella fase più attiva del movimento - dal 1854 al 1857 - il principe Luciano (e non Achille) Murat, secondogenito di Gioacchino Murat re di Napoli dal 1808 al 1815 e che si dichiarava adesso pretendente al trono occupato un tempo dal padre, accolse il sostegno di alcuni emigrati politici di parte democratica ansiosi di trovare una soluzione al problema non solo delle Due Sicilie ma dell'intera Italia. La presenza di un nuovo Napoleone sulla scena internazionale (non tanto al pretendente quanto a lui i murattiani guardavano infatti con una certa fiducia) e la crisi del Regno delle Due Sicilie portavano a vagheggiare una soluzione del problema italiano che partisse dal Meridione; concetto presente non soltanto fra i repubblicani, come prova l'interesse molto vivo suscitato all'epoca dall'alternativa murattiana in vaste aree anche di diverso orientamento politico.

(6) Lettera di Giuseppe Mazzini, 8 Settembre 1848, da Lugano, a Lizabe Ruffoni, a Parigi, in Scritti editi e inediti di Giuseppe Mazzini, vol. XXXV (Epistolario, vol. XIX); Imola, 1922; lettera di Giuseppe Mazzini, da Lugano, 9 settembre 1848, a Lizabe Ruffoni, a Parigi, in Scritti editi ecc. cit. Appendice (Epistolario, vol. IV), Imola, 1940; lettera di Giuseppe Mazzini da Losanna, 11 settembre 1849 a Lizabe Ruffoni, a Parigi, in Scritti editi, ecc. cit Appendice (Epistolario, vol. IV), Imola, 1940..

(7) Il nome di Lizabe Ruffoni ("Addio. Salutate Scipio (Scipione Pistrucci, n.d.r.) e Lizabe" ricorre ancora in una lettera indirizzata il 20 aprile 1849 da Francesco Daverio (allora capo di Stato Maggiore della Legione italiana agli ordini di Garibaldi) a Giuseppe Mazzini. Nella lettera Daverio interveniva sui temi più scottanti della politica militare della
Repubblica romana a dieci giorni dall'inizio della difesa vera e propria. La lettera è stata recentemente acquistata sul mercato antiquario dall'Istituto della Domus Mazziniana di Pisa ed è reperibile su Internet.

(8) F. BARTOCCINI, Il Murattismo, Speranze, timori e contrasti nella lotta per l'unità italiana, Milano, Giuffrè, 1959.

(9) F. BARTOCCINI, Il "Murattismo", realtà e immagine nella storia del decennio, in Correnti ideali e politiche della sinistra italiana dal 1849 al 1861. Atti del XXI Convegno storico toscano, Castelvecchio Pascoli, 26-29 maggio 1975.

(10) F. BARTOCCINI, Il Murattismo, realtà e immagine, Atti del XXI Convegno, cit., pp. 110-111.

(11) F. Bartoccini, Il Murattismo, Speranze e timori, cit. pagg. 201-202. 
Dunque, Gaetano Lizabe Ruffoni, approdato per la seconda volta in Francia, si allontana dopo alcuni anni dall'ambiente degli esuli politici repubblicani - un ambiente smarrito sotto il peso della sconfitta e della delusione e talora immiserito da pettegolezzi e rancori come può accadere anche alle personalità più nobili in situazioni che oggi definiremmo stressanti - per mettersi al servizio dal 1855, prima come bibliotecario e segretario, poi sostituendo Aurelio Saliceti alla testa del partito, del principe Luciano Murat.

Lizabe Ruffoni fu molto legato al pretendente anche sul piano personale, aiutandolo perfino nell'attività privata e impegnandosi al suo fianco nelle battaglie interne della massoneria francese. Dopo la sua "conversione" entrò spesso in feroce polemica nei confronti dei vecchi compagni mazziniani, i quali lo ripagavano con la stessa moneta, e si adoperò attivamente per la diffusione del movimento attraverso scritti propagandistici. Di lui si citano soprattutto: A ceux qui se défendent d'être muratistes ${ }^{12}$, e una lettera alla stampa dal titolo ironico Les complots muratistes ${ }^{13}$, scritti che ebbero larga risonanza e suscitarono un vivace dibattito.

Nell'agosto 1860 , mentre era in pieno svolgimento l'impresa garibaldina nell'Italia meridionale, il principe Luciano Murat ripropose la propria candidatura al trono borbonico ormai vacillante, diffondendo un ampio opuscolo dal titolo Naples et le Piémont, anch'esso dovuto probabilmente all'abile penna del Lizabe Ruffoni ${ }^{14}$. Allo stesso venne affidato poco dopo l'incarico di recarsi a Napoli per compiervi una missione esplorativa; lì pare avesse ottenuto contatti, che tuttavia risultarono improduttivi, con Alexandre Dumas padre e con Liborio Romano ${ }^{15}$. Lizabe Ruffoni aveva in precedenza indirizzato al Monitore di Bologna ${ }^{16}$ una lettera aperta nella quale ricalcava i punti del programma murattiano, sostenendo la legittimità dei diritti del pretendente al trono delle Due Sicilie.

Quando anche gli ultimi sostenitori del Murat lo abbandonarono di fronte alla prospettiva unitaria, Lizabe Ruffoni rimase a fianco del Principe, da cui si sarebbe staccato solo quattro anni dopo, da lui congedandosi ufficialmente con una lettera.

Una lettera che possiamo riprodurre - benchè solo in parte - unitamente a stralci di un'altra indirizzata, sempre dal Lizabe-Ruffoni, a Vittorio Emanuele II. Le abbiamo rintracciate in un vecchio articolo del Giornale del Mattino di Bologna: si tratta di uno scritto del 1916 dovuto a un cugino del Lizabe Ruffòni ${ }^{17}$. L'articolo, pubblicato in un periodo nel quale erano ancora presenti e vivi gli echi delle battaglie risorgimentali, e in un momento, ricordiamolo, in cui appariva doveroso fare appello ai sentimenti di unità nazionale - come puntualmente viene fatto in chiusura -è una vera e propria apologia del cugino Lizabe "molto più vecchio di me", in garbata polemica con un precedente scritto della Gazzetta Ferrarese e soprattutto con le affermazioni contenute in una vecchia opera del Canini ${ }^{18}$.

L'autore prende avvio dalla prima giovinezza del Lizabe Ruffoni, tratta degli studi compiuti dal giovane intelligente e ribelle, dei suoi primi scontri con l'autorità costituita e conseguenti imprigionamento e bastonatura per ordine del Legato pontificio: accenna al primo soggiorno in Francia, al ritorno nelle gloriose giornate del 1848, al periodo della Repubblica Romana per giungere quindi al definitivo esilio Oltralpe:

(12) G. Lizabe Ruffoni, À ceux qui se défendent d'etre muratistes, Bruxelles, Guyot, 1857

(13) Riportata nella traduzione italiana de L'Italia del Popolo del 4 settembre 1857 in F. Bartoccini, Il Murattismo, Speranze e timori, cit. pagg. 209/211.

(14) Senza data né luogo di pubblicazione, ma Parigi, agosto 1860 .

(15) S. CognetTi, Le memorie dei miei tempi,
Napoli, 1874, pag. 295.

(16) Monitore di Bologna, 25 agosto, 4 settembre 1860.

(17) G. Ruffoni, Lizabe Ruffoni, Segretario di G. Mazzini, in «Il Giornale del Mattino», Bologna, 7 settembre 1916 .

(18) M. A. Canini, Briciole di storia, Torino, 1882, pag. 78 e segg. 
affronta poi il momento critico dell'abbandono del mazzinianesimo e dell'adesione al murattismo. L'articolista esclude categoricamente a tale proposito qualsiasi interesse che non fosse di ordine ideale e morale, e ciò in base alla propria conoscenza dell'indole e del carattere del cugino Gaetano. E a riprova di ciò riproduce parzialmente il testo delle lettere che questi inviò, come abbiamo detto sopra, nel 1864 a Vittorio Emanuele II e a Luciano Murat:

\section{"Al re Vittorio Emanuele II"}

M'aggregai in Parigi nel 1855 a parecchi cittadini italiani che intesero a fare d'un mutamento di Stato e di Dinastia in Napoli, il principio e, per dir cosi, la leva di una quarta rivoluzione italiana. Per diverse vie mirai sempre allo stesso scopo, e se m'ingannai nella elezione dei mezzi, m'ingannai con uomini onorevolissimi (........ righe illeggibili) mi rivelò l'amore e la fede che a voi deve ogni vero italiano".

"Al Principe Luciano Murat":

Quello che avviene in me è la vittoria dell'amore della patria sulla mia devozione agli interessi di V.A.R. (.............)

Nato cittadino italiano, oggi convinto che il Regno d'Italia non è più l'esperienza di un santo diritto, ma un fatto certo, potente, radicato nella coscienza di una rinnovata nazione, io credo adempiere il dover mio manifestando questo maturato convincimento, e dichiarandomi suddito della vigente legge e del nuovo Re d'Italia (......).

Questo il commento dell'articolista: "Non è con un linguaggio così vibrante e solenne che si giustificano simili voltafaccia. Ė l'accento del cospiratore, dell'esule, che si sente e si confessa sempre fervidamente italiano (...). Falsa quindi ogni accusa di "salti acrobatici" dal mazzinianesimo al murattismo e da questo alla monarchia sabauda per bassi interessi venali "prescindendo dall'alterezza e onestà di mio cugino, che al lavoro e agli studi chiese sempre il pane".

Una difesa che sembra formulata in buonafede, benché sull'argomento ancora gli storici del ventesimo secolo siano piuttosto severi, in quanto riscontrano nelle attività e negli scritti del Lizabe Ruffoni "murattista" non una conversione, ma un tatticismo ondivago, non "un nuovo orientamento (...) ma un rinnegamento del passato", una sua "intelligente abilità a cogliere e a sfruttare su un piano propagandistico i motivi offerti da avvenimenti in rapida evoluzione" 19 .

Tornando all'articolo del Giornale del Mattino, l'autore accenna anche alle doti intellettuali del cugino Gaetano: "prosatore elegante, limpido, acuto, (...), buon poeta", che "per la mia laurea in giurisprudenza nel novembre 1871 mi mandò da Parigi un sonetto nel quale descriveva gli orrori della Comune". Dopo la proclamazione del Regno d'Ita1ia, Gaetano Lizabe Ruffoni che "se fosse ritornato in patria vi avrebbe ricevuto onori e gradi che ebbero altri "non superiori a lui", preferì rimanere in Francia "per l'attrattiva spirituale di quella nazione della quale scriveva magistralmente la lingua" e dove "aveva trovato una virtuosissima compagna".

Oggi ne conosciamo il nome: si chiamava Julie Antoinette De Grech, e probabilmente lo assistette negli ultimi istanti di vita nel domicilio coniugale di Parigi, rue Lamartine 16 quando, martedì 26 settembre 1876 alle sette di sera Louis Gaétan Lizabe Ruffoni, homme de Lettres nato a Ferara (sic), Italie, vi esalò l'ultimo respiro all'età di sessant'anni, come dichiarato nell'atto di morte da noi reperito presso gli Archivi dello Stato Civile di Parigi. Testimone dell'atto pubblico, oltre a certo Henri Prud'homme, maître d'bôtel, anche quell'Eugène Caimi, colonnello a riposo, la cui orazione funebre tradotta dal francese troviamo riportata nell'articolo del Giornale 
del Mattino più sopra citato: un'orazione connotata dallo stile ridondante dell'epoca ma nella quale si avverte il sincero dolore per la perdita d'un amico: pronunciata su una tomba della quale ignoriamo l'ubicazione ma che riteniamo in terra francese e probabilmente in qualcuno dei cimiteri della capitale (non certo al Père-Lachaise, ultima dimora dei potenti, dove infatti si trova l'elegante monumento dedicato a Gioacchino Murat e ai suoi).

"Ruffoni era un bel vecchio rubicondo coi capelli e i mustacchi bianchissimi, e una statura quasi colossale" aveva scritto L'Illustrazione Italiana. Vecchio a sessant'anni, secondo i criteri del diciannovesimo secolo, come il suo quasi coetaneo Flaubert che, nato nel 1821, si spegnerà nel 1880, a cinquantotto anni e mezzo. Come lui alto e rubicondo, come lui di abbondante chioma e di corporatura quasi colossale. Entrambi, fatte ovviamente le debite proporzioni, interessati alla cultura, soprattutto letteraria. Due parigini di adozione ${ }^{20}$ che non sapremo mai se abbiano potuto entrare in rapporto, magari soltanto casuale, o nelle austere sale della Grande Bibliothèque oppure sfiorandosi su un marciapiedi di qualche boulevard. Simili anche nella morte, improvvisa per entrambi. Diversi certo nella "fortuna": il primo oggetto di sterminate ricerche, il secondo confinato in un'oscurità quasi completa alla quale è stato qui possibile concedere un minuscolo spiraglio di luce.

ADRIANA SANTORO

(19) F. Bartoccini, Il Murattismo, Speranze e timori, cit. pag. 202.

(20) Gustave Flaubert, impropriamente detto "l'eremita di Croisset", pur prediligendo quella dimora nelle vicinanze della città natale (Rouen), amava in realtà soggiornare nella capitale anche per lunghi periodi. 


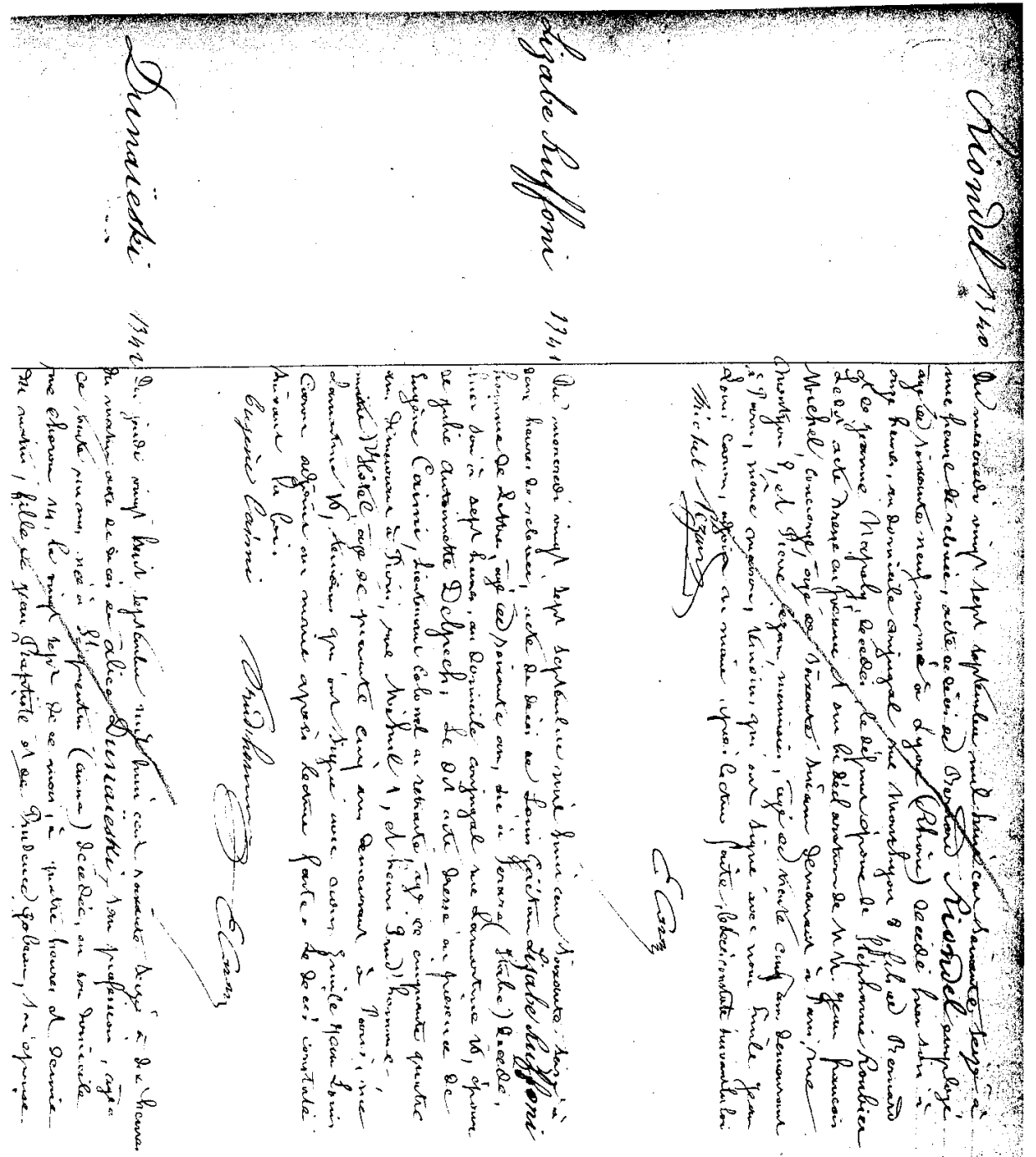

